\title{
The effect of dietary intake of antioxidant micronutrients on burn wound healing: a study in a tertiary health institution in a developing country
}

\author{
Mary Adjepong ${ }^{1}$, Pius Agbenorku², Patricia Brown ${ }^{1}$ and Ibok Oduro ${ }^{1}$
}

\begin{abstract}
Background: Burn injury results in emotional stress affecting dietary intake and antioxidant micronutrient intake, which is known to have effects on recovery outcomes. The study aimed to assess dietary intake of antioxidant micronutrients and recovery outcomes of burn patients.

Methods: Questionnaires were administered to 40 burn patients at Komfo Anokye Teaching Hospital (Ghana) from January 1, 2014 to May 30, 2014. The data taken include anthropometric measurements and dietary assessment. Their nutrient intakes were assessed with the Nutrient Analysis Template. The average intakes were compared to the recommended daily allowance. Assessment of recovery was based on records of wound healing assessments and infection rates from the health practitioners.

Results: A cross-sectional study of 40 patients revealed an average total burn surface area (TBSA) of $31.4 \%$, where 70.0, 35.0, 75.0, 52.5, 12.5 and $32.5 \%$ patients were deficient in vitamins $A, C$ and E, zinc, copper and selenium, respectively and adequate amounts of vitamin $C$ intake were related with significantly better wound healing progress. Positive wound healing outcomes were observed for patients with adequate vitamins $A$ and $E$ and zinc intake. Less infection presented among patients with adequate amount of vitamins $\mathrm{A}$ and $\mathrm{C}$ and zinc, but this was not observed for patients with adequate vitamin E, copper and selenium.

Conclusions: Most burn patients did not meet their dietary requirements for antioxidant micronutrients and this was due to meals not tailored to suit individual requirements. Adequacy of the antioxidants especially vitamin $C$ resulted in positive wound healing outcomes. Hence, there is need for planned well-balanced meals of high vitamin C.
\end{abstract}

Keywords: Dietary intake, Antioxidants, Micronutrients, Recovery, Wound infection, Wound healing

\section{Background}

Burns place a great socio-economic burden on individuals, their families and the health services [1]. Apart from the financial burden to families, communities, and the nation, it leads to pain, infection, extensive scarring, wound and scar contractures, amputations and death in some of its victims [2].

\footnotetext{
* Correspondence: pimagben@yahoo.com

${ }^{2}$ Reconstructive Plastic Surgery \& Burns Unit, Department of Surgery, Komfo Anokye Teaching Hospital, School of Medical Sciences, College of Health Sciences, Kwame Nkrumah University of Science \& Technology, Kumasi, Ghana

Full list of author information is available at the end of the article
}

The World Health Organization (WHO) estimates that 43,000 people die of burns in Africa every year with a rate of 6.1 per 100,000 [2]. In Ghana, the incidence is 6.1 per 100,000 persons, an extrapolated incidence of $1.3 \%$ with more women and children being victims because of the roles of a typical Ghanaian woman. Burn injury accounts for 265,000 deaths annually and children below 20 are the most affected. It is important to note that majority of deaths occur in low- and middle-income countries [3].

The main causes of mortality in burn patients are associated with infection, delayed wound healing, and 
extensive protein catabolism. Underlying these processes include immunological, endocrine, inflammatory and other metabolic responses [4]. When the skin is exposed in burn patients, there are several mechanisms that lead to oxidative damage to the cell membranes. This includes the peroxidation of polyunsaturated fatty acid residues in membranes that can lead to membrane impairment, in addition to fragmentation of proteins at vulnerable points in the amino acid chain. There is also the irreversible damage to protein sites where metal ions normally bind to functional protein [5].

Apart from this destruction to the cell membrane, there are several metabolic responses that render the individual susceptible to malnutrition. Among them is hypercatabolism, which contributes to the breakdown of muscle and fat. Hypermetabolic response in the individual leads to an increase in the basal metabolic rate to about $180 \%$ and an increase in the utilization of nutrients [6] and can last at least 9 to 12 months [7]. The increase in the production of catecholamines contributes to an increase in the resting energy expenditure. Similarly, the release of glucocorticoids leads to an alteration in protein and carbohydrate metabolism. These result in an increase glucagon/insulin ratio leading to hyperglycemia. This is a result of increased gluconeogenesis, proteolysis, glycogenolysis and lipolysis [6]. There is also nutrient loss in urine and wound exudates and this can lead to hypoalbumenuria [8].

Being a central part of post burn management, medical nutrition therapy ensures adequate nutrients for the victim to improve wound healing, to restore cutaneous losses and to manage infections [9]. Apart from the role of macronutrients in these, the role of micronutrients cannot be ignored especially antioxidant micronutrients.

Copper, zinc, selenium, and vitamins $\mathrm{A}, \mathrm{C}$ and $\mathrm{E}$ are antioxidant micronutrients that are beneficial to burn patients when intakes are adequate. This is due to their roles in scavenging free radicals in the wound healing process-homeostasis, inflammatory phase, proliferative phase and remodeling - as well as their role in fighting infections $[10,11]$.

The aim of this study therefore was to assess the effect of dietary intake of antioxidant micronutrients on the various recovery outcomes of burn patients. The micronutrients considered were copper, zinc, selenium and vitamins $\mathrm{A}, \mathrm{C}$ and $\mathrm{E}$. The recovery outcomes are the progress of wound healing, presence of infection and the length of stay in the hospital.

\section{Methods}

\section{Study setting}

The Komfo Anokye Teaching Hospital is the second largest hospital in Ghana. It is the main referral hospital for the northern sector of Ghana. With a bed capacity of over a thousand, there are numerous units in the hospital that provide health care for persons who come to the facility. Among the units are the Reconstructive Plastic Surgery and Burns Unit under the Directorate of Surgery. In this Unit is a Burns Intensive Care Unit (BICU) that caters to burn patients who are critically ill. There are other wards that cater to less severe cases.

\section{Subjects}

The chosen subjects were burn patients who were admitted to the BICU from January to May 2014 of all ages who consented to the study. Some other cases of interest were also taken from the outpatient department.

\section{Data collection}

Pretested questionnaires were administered to clinically consenting patients. Demographics, medical history, anthropometry, food frequency, and triplicate dietary recalls were done. Anthropometric measurements include weight, height, and mid-upper arm circumference for children. Multiple dietary recalls were done for all patients for 3 days including a day in a weekend. On admission, patients were fed with meals provided by the hospital.

Using handy measures, the weights of all the foods consumed by the subjects were recorded. The nutrient compositions in the foods were analyzed using the $\mathrm{Nu}$ trient Analysis Template (University of Ghana, Food Science and Nutrition Department). The average of the intakes was recorded and compared to the recommended daily intakes (University of Ghana, Food Science and Nutrition Department) of the individuals. Assessment of recovery was based on records of wound healing assessments, infection rates and the length of stay in the hospital from the health practitioners and a tape measure was also used to measure the diameter of the wounds.

\section{Data analysis}

Data storage was done using Microsoft Excel 5.0 and graphs were drawn using Microsoft Excel. Chi-square test was used to compare the categorical parameter/ qualitative variable among the groups and $p<0.05$ was considered statistically significant.

\section{Ethical clearance}

Ethical clearance was granted by the Committee of Human Research Publication and Ethics of Komfo Anokye Teaching Hospital (KATH)/School of Medical Sciences, Kwame Nkrumah University of Science and Technology (KNUST), Kumasi. 


\section{Results}

Demographic data

In Table 1, the demographic statistics of burn patients at KATH from January to May 2014 is shown. Out of the 40 patients, 23 of them were aged above 14 years representing the highest age range followed by those who were aged between 2 and 14 years with 9 subjects. The lowest reported age range was for patients below 2 years. The results also showed more males suffering from burn injury (65 \%) than females (35\%). Only two subjects, representing a total of $5 \%$, had a level of tertiary education, with majority of the subjects having no education (30\%), primary level of education (37.5\%), and secondary education $(27.5 \%)$. In view of this, it was not surprising that $5 \%$ of the subjects were professionals in their fields with the rest being artisans (25\%) and traders (22.5\%) and a majority being unemployed (47.9\%).

\section{Anthropometric and medical information of the patients}

Majority of the patients (adults) in this study had normal basal metabolic rate, with fewer $(7.5 \%)$ of them being obese. The major cause of burn injury in this study was thermal causes: scalds, open flame, and explosions. Chemical and electric causes recorded minimum injury, 2.5 and $5.0 \%$, respectively. Half (50.0\%) of the patients

Table 1 Demographic profiles of 40 burn patients at KATH BICU

\begin{tabular}{lll}
\hline Characteristics & \multicolumn{2}{l}{ Number of patients } \\
\cline { 2 - 3 } & $N$ (total = 40) & $\%$ \\
\hline Age (years) & 8 & 20.0 \\
$<2$ & 9 & 22.5 \\
$2-14$ & 23 & 57.5 \\
$>14$ & & \\
Sex & 26 & 65.0 \\
Male & 14 & 35.0 \\
Female & & \\
Level of education & 15 & 37.5 \\
Primary school & 8 & 20.0 \\
Middle/junior high school & 3 & 7.5 \\
Senior high school & 1 & 2.5 \\
Tertiary 1 to 3 years & & \\
(diploma/certificate/professional) & 1 & 2.5 \\
Tertiary 4 or more years & & \\
(degree/postgraduate/professional) & & 30.0 \\
None & 12 & 25.0 \\
Occupation & & \\
Artisan & 10 & \\
Professional & & \\
Trader & & \\
Unemployed & & \\
\hline
\end{tabular}

suffered severe injury of above $25.0 \%$ total burn surface area (TBSA), followed by 10-25\% TBSA with $27.5 \%$ of the patients. In addition, majority of the patients (50\%) suffered partial thickness burns. The results are shown in Table 2.

\section{Adequacy and inadequacy of dietary antioxidant micronutrients}

When dietary intake of antioxidant micronutrients was assessed and compared to the recommended dietary allowances of the population based on the ages, the following results were obtained: $30.0 \%$ of the patients had adequate vitamin $\mathrm{A}$ and $70.0 \%$ had inadequate intakes in their diet, $65 \%$ had adequate intakes of vitamin C with $35.0 \%$ having inadequate intakes and $25.0 \%$ of the patients had adequate vitamin $\mathrm{E}$ intakes with $75.0 \%$ recording inadequate intakes.

With regard to trace element intake in the diet, the following results were recorded: 47.5, 90.0 and $67.5 \%$ had adequate dietary intake of zinc, copper, and selenium, respectively, with $52.5,12.5$, and $32.5 \%$ of the patients recording inadequate dietary intake (Table 3 ).

\section{Dietary intake of vitamin A and wound healing progress}

The measure of the recovery outcome considered was the length of hospital stay and the presence of infections. The length of stay in the hospital was dependent on the

Table 2 Anthropometric and medical information of patients

\begin{tabular}{lll}
\hline Characteristic & \multicolumn{2}{l}{ Number of patients } \\
\cline { 2 - 3 } & $N=40$ & $\%$ \\
\hline Description of basal metabolic index (BMI) & & \\
Underweight & 0 & 0 \\
Normal & 13 & 32.5 \\
Overweight & 7 & 17.5 \\
Obese & 3 & 7.5 \\
Cause of burn & & \\
Electrical & 2 & 5.0 \\
Chemical & 1 & 2.5 \\
Thermal & 37 & 92.5 \\
Total burn surface area (TBSA) & & \\
$<10 \%$ & 9 & 22.5 \\
10-25 \% & 11 & 27.5 \\
$>25 \%$ & 20 & 50.0 \\
Severity of burn & & \\
Superficial thickness & 12 & 30.0 \\
Second degree/partial thickness & 20 & 50.0 \\
Third degree/full thickness & 3 & 7.5 \\
Mixed thickness & 5 & 12.5 \\
\hline
\end{tabular}

${ }^{a}$ Mid-upper arm circumference (MUAC) was the nutritional assessment tool for children (14 years and below); all the children were within normal ranges 
Table 3 Dietary intake of antioxidant micronutrients and wound healing progress

\begin{tabular}{|c|c|c|c|c|c|c|c|}
\hline & \multirow[t]{2}{*}{$\begin{array}{l}N \\
\text { (total }=40)\end{array}$} & \multicolumn{3}{|c|}{$\begin{array}{l}\text { Week } 1 \text { wound } \\
\text { healing }\end{array}$} & \multicolumn{3}{|c|}{$\begin{array}{l}\text { Week } 2 \text { wound } \\
\text { healing }\end{array}$} \\
\hline & & $\bar{n}$ & $(\%)$ & $p$ value & $\bar{n}$ & $(\%)$ & $p$ value \\
\hline \multicolumn{8}{|l|}{ Vitamin A } \\
\hline Adequate & 12 & 10 & (83) & 0.162 & 7 & $(58)$ & 0.629 \\
\hline Inadequate & 28 & 17 & $(61)$ & & 14 & $(50)$ & \\
\hline \multicolumn{8}{|l|}{ Vitamin C } \\
\hline Adequate & 26 & 21 & $(81)$ & 0.015 & 16 & $(62)$ & 0.119 \\
\hline Inadequate & 14 & 6 & $(43)$ & & 5 & (36) & \\
\hline \multicolumn{8}{|l|}{ Vitamin E } \\
\hline Adequate & 9 & 7 & (78) & 0.455 & 5 & (56) & 0.835 \\
\hline Inadequate & 31 & 20 & $(65)$ & & 16 & $(52)$ & \\
\hline \multicolumn{8}{|l|}{ Zinc } \\
\hline Adequate & 19 & 15 & (79) & 0.141 & 11 & (58) & 0.516 \\
\hline Inadequate & 21 & 12 & (57) & & 10 & $(48)$ & \\
\hline \multicolumn{8}{|l|}{ Copper } \\
\hline Adequate & 36 & 24 & $(67)$ & 0.736 & 18 & $(50)$ & 0.342 \\
\hline Inadequate & 4 & 3 & $(75)$ & & 3 & (75) & \\
\hline \multicolumn{8}{|l|}{ Selenium } \\
\hline Adequate & 26 & 19 & (73) & 0.305 & 13 & $(50)$ & 0.666 \\
\hline Inadequate & 14 & 8 & (57) & & 8 & (57) & \\
\hline
\end{tabular}

$p<0.05$ was considered statistically significant

TBSA of the patient. The study of the wound healing outcomes for 2 weeks revealed that out of the 12 patients who had adequate dietary intake of vitamin A, $83.3 \%$ of them had a positive wound healing outcome during the first week with $58.3 \%$ of the patients recording positive wound healing outcomes in the second week. When this is compared to 28 patients with inadequate dietary intake of vitamin $\mathrm{A}$, it can be observed that 60.7 and $50.0 \%$ of the patients had positive wound healing outcomes, showing a decrease in the wound healing outcomes. When they were compared statistically, the $p$ values showed that there was no statistical difference in the outcomes in weeks, 0.162 and 0.629 when patients with adequate intake and inadequate intake were compared.

\section{Dietary intake of vitamin $\mathrm{C}$ and wound healing progress}

Similarly, the wound healing outcomes for patients with adequate intake of vitamin $C$ showed a remarkable progress in wound healing. Out of 26 patients, 80.8 and $61.5 \%$ of them recorded positive wound healing outcomes for weeks 1 and 2, respectively. In contrast, there was progress in wound healing for only 42.9 and $35.7 \%$ of the patients with inadequate dietary intake of vitamin $\mathrm{C}$ for the first and second weeks, respectively. Also, there was statistical significance between vitamin $\mathrm{C}$ intake and wound healing in week 1 with a $p$ value of 0.015 when patients with adequate intake and inadequate intake were compared.

\section{Dietary intake of vitamin $E$ and wound healing progress}

The role of vitamin $E$ in the progress of wound healing is outstanding. It was observed that the 9 patients with adequate intake of vitamin $\mathrm{E}$ recorded positive outcomes of 77.8 and $55.6 \%$ in wound healing in the first and second weeks, respectively, as compared to the $31 \mathrm{pa-}$ tients with inadequate dietary intake of the vitamin who recorded 64.5 and $51.6 \%$ in their wound healing outcomes. When they were compared statistically, the $p$ values showed that there was no statistical difference in the outcomes in weeks, 0.455 and 0.835 when patients with adequate intake and inadequate intake were compared.

\section{Dietary intake of zinc and wound healing progress}

There was a remarkable wound healing progress in the patients who had adequate dietary intake of zinc. There was a corresponding increase in the number of patients with regard to their wound healing outcomes as seen in the previous antioxidants; 79.0 and $57.9 \%$ of the patients recorded positive progress in wound health out of the 19 patients who had adequate dietary intake of the trace element as compared to those with inadequate dietary intake of the trace element who recorded 57.1 and $47.6 \%$ in their wound healing outcomes. When they were compared statistically, the $p$ values showed that there was no statistical difference in the outcomes in both weeks, 0.141 and 0.516 when patients with adequate intake and inadequate intake were compared.

\section{Dietary intake of copper and wound healing progress}

The adequacy in the dietary intake of copper also yielded remarkable wound healing outcomes. The first and second weeks recorded a positive wound healing rate of 66.7 and $50.0 \%$ for the 36 patients with adequate dietary intake of copper. Patients with inadequate amounts of this trace element in their diet recorded a relatively higher progress in the wound health outcomes recording $75.0 \%$ for each week. When they were compared statistically, the $p$ values showed that there was no statistical change in the outcomes in both weeks, 0.736 and 0.342 when patients with adequate intake and inadequate intake were compared.

\section{Dietary intake of selenium and wound healing progress}

The adequacy of selenium in the diet of patients follows the same trend as the other antioxidant nutrients. There was a progress in the wound healing outcomes in the first and second weeks for patients who had adequate intake of selenium in their diet. It was also recorded that 
there was a relative decrease in the number of patients who had inadequate amounts of selenium in their diet. When they were compared statistically, the $p$ values showed that there was no statistical change in the outcomes in both weeks, 0.305 and 0.666 when patients with adequate intake and inadequate intake were compared.

\section{Adequacy of antioxidant vitamins and infection}

The rate of wound infection in patients with adequate dietary intake of vitamin A was $25.0 \%$ compared to patients who had inadequate dietary intake recording a relatively higher figure, $32.0 \%$. Patients with adequate dietary intake of vitamin $\mathrm{C}$ also recorded a lower rate (26.9\%) of infections compared with patients with inadequate dietary intake $(35.7 \%)$. Though there were contrasting results, patients with adequate vitamin $\mathrm{E}$ recorded a wound healing rate of $44.4 \%$ compared with their counterparts with inadequate amounts recording a lower rate of infection (24.8 \%) as shown in Fig. 1.

\section{Adequacy of antioxidant trace elements and infection}

Patients with adequate dietary intake of zinc recorded a lower rate of infection (26.3\%) as compared to patients with inadequate amounts. With regard to copper and selenium, the infection rate was relatively lower in patients with inadequate amounts (Fig. 2).

\section{Discussion}

Children aged 14 and below are known to have a high incidence of burn injury in developing countries, and this could be a result of the roles played by most children with their mothers in the household [12]. Though children in this category did not record the highest figure; they recorded a considerably high percentage $(42.5 \%)$ of the total number of patients, giving the impression that the child within this category needs to be protected from burn injury. Also, children below age 2 years recorded $20.0 \%$ for the burn injury which is quite high for such an age group. This could be as a result of negligence of mothers as well as the exposure of children to open flames in a typical setting of a home in a developing country [13]. Also, the socio-economic background has an effect on burn injury. Only $5.0 \%$ of the participants of this study belonged to a high socioeconomic class corresponding to works done by Bell et al. [14] which states that burn injury increases substantially with a lower socio-economic status.

The major cause of burn in this study was thermal (92.5\%) comprising of open flames, scalds, and gas explosion with chemical (2.5\%) and electrical (5.0 \%) causes. This corresponds to the works done by Agbenorku et al. [15]. The results from this study also show that majority (50.0\%) of the patients suffered burns with a TBSA above $25.0 \%$ which is quite alarming because most fatalities occur in such severities. In addition, $70.0 \%$ of the participants had burn injury of second degree, third degree, and mixed thickness, giving an indication that most of the injuries were very severe. In their study, Gokdemir et al. [16] reported that mortality increases with increasing TBSA and burn depth. Also, the presence of ascobate in leukocytes and its rapid

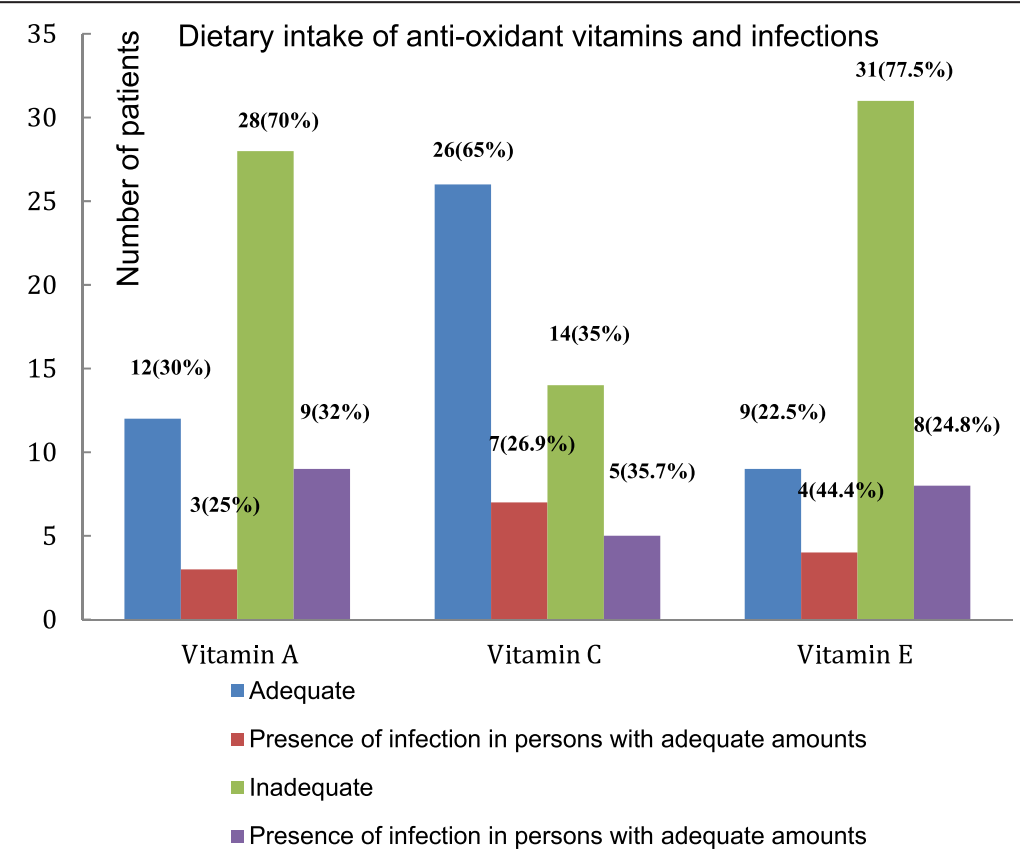

Fig. 1 Intake of antioxidant vitamins and infection 


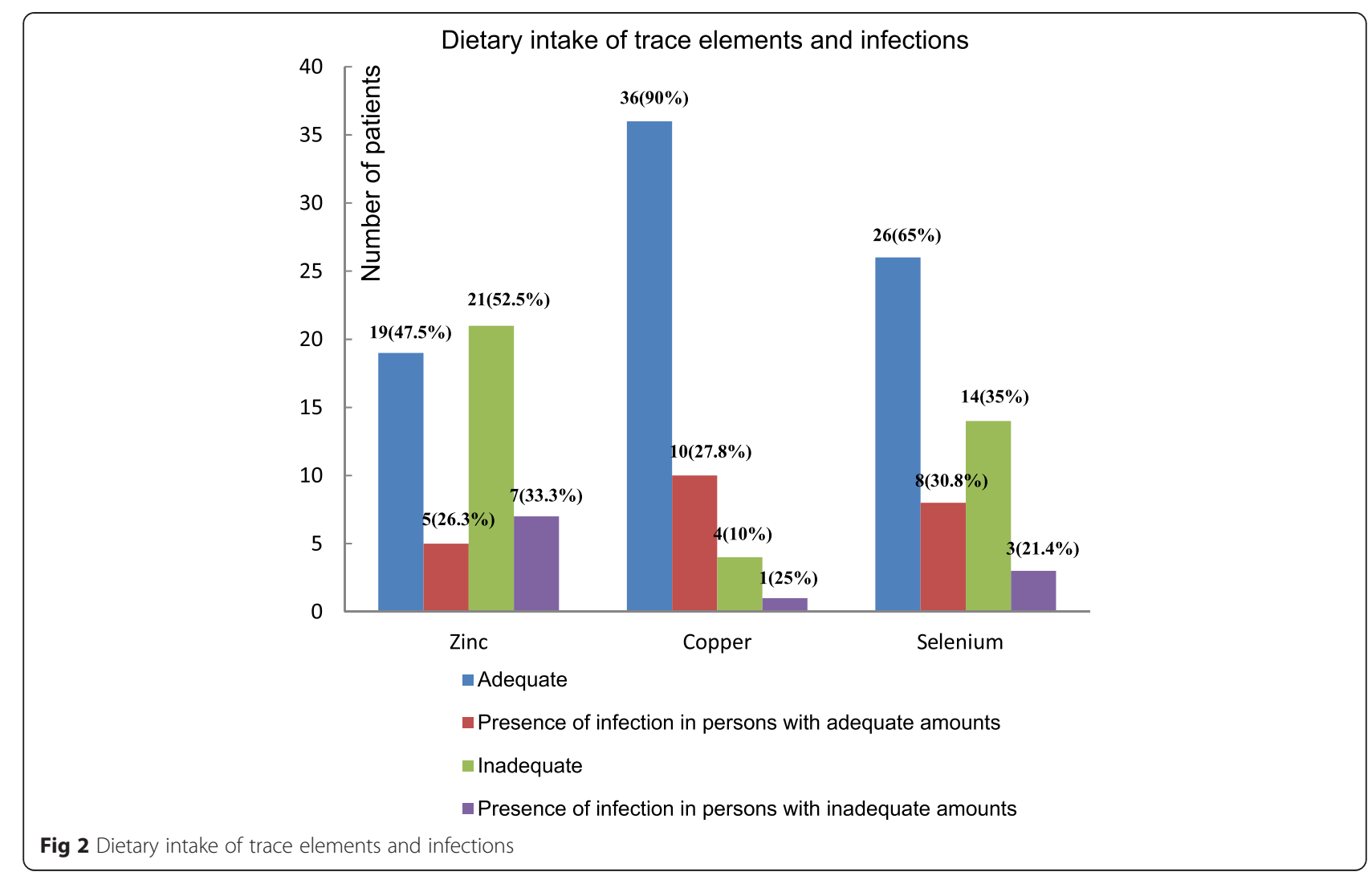

expenditure during infection and phagocytosis suggest that vitamin $\mathrm{C}$ has a role in immunity, hence the results obtained.

Vitamin C (ascorbic acid) influences all phases of wound healing such as the inflammatory phase, proliferative phase, and maturation, synthesis, and degradation of collagen [10]. Collagen synthesis, maturation and stabilization are also roles of vitamin C. It is also responsible for neutrophil migration to the site of injury and has a role in clotting when the skin is exposed. From this study, it was noted that during the first 2 weeks of monitoring the patients, patients with adequate dietary intake of vitamin $\mathrm{C}$ recorded a remarkable increase in wound healing. This corresponds to the work done by Barbosa et al. [17] and Sahib et al. [18] where supplementation of vitamin $\mathrm{C}$ reduced wound healing time. The results also revealed a significant change with a $p$ value of 0.015 in the first week indicating that there was a significant improvement in wound healing outcomes in the first week.

Vitamin A is known to play diverse roles in the wound healing cascade. It enhances early inflammatory phase and promotes epithelial cell differentiation, collagen deposition, and immunity [10]; hence, patients with adequate dietary intake had progress in wound healing during the first 2 weeks. Also, intake of vitamin A results in an increase in lymphocyte proliferation, and this has the ability to prevent wound infection due to the reduction of microbes on wounds. Vitamin A is also known to aid in the repair of damaged tissue and hence may be beneficial in counteracting free radical damage [19], increasing the wound healing rate.

When a wound is exposed, the presence of microorganisms greater than 100,000 results in no epithelialization. Vitamin $E$ is responsible for the preservation of macrophages which act by eliminating the microorganism by phagocytosis [10]. Due to its role in immunity, it is not surprising that patients with adequate amount in their diet had a good wound healing outcome as compared to patients with inadequate amounts in their diet. This is similar to works done by Barbosa et al. [17] and Sahib et al. [18].

The effect of adequate intake of trace element supplementation on the recovery of burn patients is noted in most studies. This is evidenced by reduced length of stay in hospitals, increase in protein turnover, as well as a reduction in infection. The body's antioxidant defense network is premiered by glutathione peroxidase, and selenium is known to be a major component of glutathione transferase enzyme, an important enzyme for this antioxidant [19]. Though the roles of these are not fully known, their role on various recovery outcomes is prominent. Copper and zinc are also co-factors of superoxide dismutase enzyme [20]. The results obtained from this 
study revealed that patients who had adequate zinc, copper, and selenium in their diet had positive wound healing outcomes and patients with adequate zinc had fewer reports of infections. This corresponds with works by Berger et al. $[4,21]$.

\section{Conclusions}

Most burn patients did not meet their dietary requirements for antioxidant micronutrient and this was due to meals not tailored to suit individual requirements, hence the need for planned and well-balanced meals. Routine supplementation of antioxidant micronutrients can also be considered. Policies to provide meals to burn patients on the ward are worth noting: vitamin $\mathrm{C}$ is the only micronutrient that showed a promising significant value (0.015) with respect to wound healing outcomes; hence, it is recommended that food with high vitamin $C$ such as fruits and vegetables should be distributed to burn patients on admission.

\section{Competing interests}

The authors declare that they have no competing interests.

\section{Authors' contributions}

MA conceived and designed the study, acquired data, and wrote and critically edited the manuscript. PA conceived and co-designed the study, analyzed and interpreted the data, and critically edited the manuscript. PB and $\mathrm{IO}$ conceived and co-designed the study and interpreted the data. All authors read and approved the final manuscript.

\section{Acknowledgements}

The authors are grateful to the entire staff of BICU and the other burn wards of the Komfo Anokye Teaching Hospital, Kumasi, Ghana, for their tremendous assistance during this research.

\section{Author details}

${ }^{1}$ Department of Food Science and Technology, College of Science, Kwame Nkrumah University of Science \& Technology, Kumasi, Ghana. ${ }^{2}$ Reconstructive Plastic Surgery \& Burns Unit, Department of Surgery, Komfo Anokye Teaching Hospital, School of Medical Sciences, College of Health Sciences, Kwame Nkrumah University of Science \& Technology, Kumasi, Ghana.

Received: 23 July 2015 Accepted: 24 July 2015

Published online: 12 August 2015

\section{References}

1. Balseven-Odabaşı A, Tümer AR, Keten A, Yorgancı K. Burn injuries among children aged up to seven years. Turk J Pediatr. 2009;51(1):328-35.

2. Outwater AH, Ismail H, Mgalilwa L, Temu MJ, Mbembati NA. Burn injuries in Tanzania: morbidity and mortality, causes and risk factors: a review. Int J Burn Trauma. 2013;3(1):18-29.

3. World Health Organization. Burn, Media centre, fact sheets, assessed May 7, 2014. 2014

4. Berger MM, Spertini F, Shenkin A, Wardle C, Wiesner C, Schindler C, et al. Trace element supplementation modulates pulmonary infection rates after major burns: a double-blind, placebo-controlled trial. Am J ClinNutr. 1998:68(1):365-71.

5. Webb GP. Dietary supplements and functional foods. 2nd ed. New York: Wiley Blackwell; 2011.

6. Williams FN, David N, Herndon DN, Jeschke MJ. The hypermetabolic response to burn injury and interventions to modify this response. Clin Plast Surg. 2009;36(4):583-96.

7. Prelack K, Dylewski M, Sheridan RL. Practical guidelines for nutritional management of burn injury and recovery. Burns. 2007;33(1):14-21.
8. Al-Jawad F, Sahib AS. Effect of N-acetylcysteine on wound healing in burned patients. Mustansiriya Medical J. 2011;10(1):28-31.

9. Prins A. Nutritional management of the burn patient. South African J Clinical Nutrition. 2009;22(1):9-15.

10. MacKay D, Miller AL. Nutritional support for wound healing. Altern Med Rev. 2003;8(2):359-77.

11. Chianeh RY, Rao P. Role of copper and vascular endothelial growth factor (VEGF) on endometrial angiogenesis. J Krishna Institute of Medical Sciences University. 2013;2(2):6-17.

12. Brusselaers N, Monstrey S, Vogelaers D, Hoste E, Blot $\mathrm{S}$. Severe burn injury in Europe: a systematic review of the incidence, etiology, morbidity, and mortality. Critical Care 2010; 14(1). doi: 10.1186/cc9300.

13. Rayner R, Prentice J. Paediatric burns: a brief global review. Wound Practice and Res. 2011;19(1):39-46.

14. Bell NJ, Schuurman N, MoradHameed S. A small-area population analysis of socioeconomic status and incidence of severe burn/fire-related injury in British Columbia. Canada Burns. 2009;35(1):1133-41.

15. Agbenorku P, Akpaloo J, Yalley D, Appiah A. A new era in the management of burns trauma in Kumasi, Ghana. Annals of Burns and Fire Disasters. 2010;23(2):59-66.

16. Gokdemir MT, Mustafa AM, Sogut O, Cahfer GC, Sayhan MB, Murat Orak M, et al. Clinical outcome of patients with severe burns, presenting to the emergency department. J Current Surgery. 2012;2(1):17-23.

17. Barbosa E, Faintuch J, Machado M, da Silva VR G. Supplementation of vitamin $E$, vitamin $C$, and zinc attenuates oxidative stress in burned children; a randomized, double blind, placebo-controlled pilot study. J Burn Care Res. 2009;30(5):859-66.

18. Sahib AS, Al-Jawad F. Effect of antioxidants micronutrients on the incidence of wound infection in burn patients. Annals of Burns and Fires Disaster. 2010;23(4):199-205.

19. Nooris S. An overview of oxidative stress and antioxidant defence system, Open Access Scientific Reports, 2008: 1(8). doi:10.4172/scientific reports, 413.

20. Tiingi U. Selenium, its role in human health. Environ Health Prev Med. 2008;13(2):102-8.

21. Berger MM, Eggimann P, Heyland DK. Reduction of nosocomial pneumonia after major burns by trace element supplementation: aggregation of two randomised trials. Crit Care. 2006;10(6):R153. doi:10.1186/cc5084.

\section{Submit your next manuscript to BioMed Central and take full advantage of:}

- Convenient online submission

- Thorough peer review

- No space constraints or color figure charges

- Immediate publication on acceptance

- Inclusion in PubMed, CAS, Scopus and Google Scholar

- Research which is freely available for redistribution 